\title{
On the use of area-averaged void fraction and local bubble chord length entropies as two- phase flow regime indicators
}

\author{
Leonor Hernández ${ }^{1}$, J. Enrique Juliá ${ }^{1, *}$, Sidharth Paranjape ${ }^{2}$, Takashi Hibiki ${ }^{2}$, \\ Mamoru Ishii ${ }^{2}$ \\ ${ }^{1}$ Universitat Jaume I. Departamento de Ingeniería Mecánica y Construcción \\ Campus de Riu Sec, Castellón, E-12071, Spain. \\ ${ }^{2}$ Purdue University. Nuclear Engineering Department West Lafayette, Indiana, \\ USA
}

\section{ABSTRACT}

In this work, the use of the area-averaged void fraction and bubble chord length entropies is introduced as flow regime indicators in two-phase flow systems. The entropy provides quantitative information about the disorder in the area-averaged void fraction or bubble chord length distributions. The CPDF (cumulative probability distribution function) of void fractions and bubble chord lengths obtained by means of impedance meters and conductivity probes are used to calculate both entropies. Entropy values for 242 flow conditions in upward two-phase flows in $25.4 \mathrm{~mm}$ and $50.8 \mathrm{~mm}$ pipes have been calculated. The measured conditions cover ranges from $0.13 \mathrm{~m} / \mathrm{s}$ to $5 \mathrm{~m} / \mathrm{s}$ in the superficial liquid velocity $j_{f}$ and ranges from $0.01 \mathrm{~m} / \mathrm{s}$ to $25 \mathrm{~m} / \mathrm{s}$ in the superficial gas velocity $j_{g}$. The physical meaning of both entropies has been interpreted using the visual flow regime map information. The area-averaged void fraction and bubble chord length entropies capability as flow regime indicators have been checked with other statistical parameters and also with different input signals durations. The area-averaged void fraction and the bubble chord length entropies provide better or at least similar results than those obtained with other indicators that include more than one parameter. The entropy is capable to reduce the relevant information of the flow regimes in only one significant and useful parameter. In addition, the entropy computation time is shorter than the majority of the other indicators. The use of one parameter as input also represents faster predictions.

Keywords: two-phase flow, flow regime identification, neural networks, impedance meter, conductivity probe, two-phase flow entropy

* Corresponding author: Phone: +34964728139, Fax: +34964728106, e-mail: bolivar@emc.uji.es 


\section{INTRODUCTION}

Gas-liquid two-phase flows are frequently encountered in important industrial applications such as boilers, nuclear power plants, petroleum transportation and various types of chemical reactors. The two phases can flow according to several topological configurations called flow patterns or flow regimes, which are determined by the interfacial structure between both phases. The existence of a particular flow regime depends on a variety of parameters, which include the properties of the fluids, the flow channel size, geometry and orientation, body force field and flow rates.

Vertical two-phase flows are usually classified into four or five basic flow regimes (Taitel et al., 1980; Mishima \& Ishii; 1984). A gradual increment in the gas flow rate consecutively produces the transition between different flow patterns when an upward two-phase flow in a vertical pipe with a constant liquid flow rate is considered. Therefore, bubbly (B), cap-bubbly (CB), slug (S), churn-turbulent (CT) and annular (A) flow patterns can be found (Figure 1).

The identification of the flow regimes and their transitions is important for industrial application as well as from the theoretical analysis point of view. On one hand, almost all constitutive relations in a two-fluid model (Ishii and Hibiki, 2007; Ishii \& Mishima, 1984) depend on the flow regime since physical mechanisms vary with flow regime transitions. Additionally, industrial equipment offers durability and safety only when the installation operates according to the flow regimes that it was designed for because important parameters, such as heat and mass transfer rates, pressure drop, etc, are directly connected with the flow regime.

Flow regime definitions are based on linguistic descriptions and graphical illustrations like those in Figure 1, and therefore, their identification is strongly subjective. Many researchers have been working on developing objective flow regime identification methodologies. Most flow regime identification approaches have two steps in common: the first step consists of developing an experimental methodology for measuring certain parameters that are intrinsic to the flow and are also suitable flow regime indicators. In the second step, a non-linear mapping is performed to obtain an objective identification of the flow regimes in accordance with these indicators. There are two important parameters to characterize the two-phase flow geometry, namely, void fraction and interfacial area concentration. Consequently, the flow regime indicators have to be related with such parameters. In this regard, void fraction distributions obtained with differential pressure transducers (Jones and Zuber, 1975; Tutu 1982; Matsui 1984) or non-intrusive impedance void metes (Mi et al., 1998; 2001a) have been widely used as flow regime indicators in the last decades. In this case the flow regime is defined as a global, 3D or 2D, parameter (Global Flow Regime, GFR). Recently, Julia et al. (2008) used bubble chord length distributions obtained from conductivity probes as flow regime indicators. 
Bubble chord length represents the bubble size and is related with the interfacial area concentration. Thus, the flow regimes are defined as time-averaged bubble chord length patterns and they are considered as local parameters (Local Flow Regimes, LFR). The main advantages of this approach over the conventional flow regime identification methods are the stronger physical relevance of the bubble chord length distribution as flow regime discriminator and, thus, the reduction in the temporal length of the signal needed to obtain reliable results. In addition, the identification of LFR in different radial locations provides $2 \mathrm{D}$ local flow regime maps that can be used to identify new GFR configurations, not available with the conventional identification methods. Finally, the experimental apparatus needed for the identification (conductivity probe) is simple, affordable and commonly used in two-phase flow characterization.

In the first flow regime identification works the flow regime mapping were carried out directly by the researcher. A significant advance in the objective flow regime mapping was achieved by the use of artificial neural networks (ANN) (Cai et al., 1994; Mi et al. 1998; 2001a, 2001b). Using statistical parameters from the void fraction distribution and Kohonen Self-Organizing Neural Networks (SONN) it was possible to identify the flow regimes more objectively. Subsequently, some improvements in the methodology developed by Mi et al. have been made. Lee et al. (2008a) used the Cumulative PDF (CPDF) of the impedance void meter signals as the flow regime indicator. The CPDF is a more stable parameter than the PDF because it is an integral parameter. Also, it has a smaller input data requirement that makes faster flow regime identification possible. Hernandez et al. (2006) developed different neural network strategies to improve the flow regime identification results. Different types of neural networks, training strategies and flow regime indicators based on the CPDF were tested in their work. In order to minimize the effect of the fuzzy flow regime transition boundaries on the identification results, a committee of neural networks was assembled. Then the identification result was obtained by averaging the results provided by all the neural networks forming the committee. In particular, the flow regime prediction capabilities of the Probabilistic Neural Networks (PNN) were verified by Sharma et al. (2006) in round pipes. In their work, a large set of experimental data obtained in round pipes was used for training a PNN using the superficial velocities, pipe diameter and inclination angle as inputs. The results given by the PNN showed good agreement even in the flow regime boundaries.

More sophisticated flow regime indicators are also available. Ruzicka et al. (1997) used the Kolmogorov entropy from pressure signals in order to discriminate between flow regimes in a bubble column. Furthermore, Zhang and Shi (1999) developed a methodology that could be used to identify flow regimes in a two-phase flow loop using the Shannon entropy of the PSD of pressure oscillation signals in a two-phase flow loop. In addition, Elperin and Klochko (2002) used the wavelet transform in order to identify two-phase flow regimes. In this case, the basis of the wavelet decomposition was the entropy and the sparsity of differential pressure transducers signals. Finally, Lee et al. (2008b) used the chaotic characteristics of time sequential impedance probe signals in 
order to identify flow regimes in upward vertical two-phase flows

In this work, the use of the area-averaged void fraction and local bubble chord length entropies as two-phase flow regime indicators is explored. The physical content of both indicators and its dependence with superficial liquid and gas velocities is analyzed. In addition, the capability of both entropies as flow regime indicators using different signal durations has been compared with other statistical parameters used in previous works.

\section{Experimental Facility and Methodology}

\subsection{Vertical two-phase flow loop}

Figure 2 shows the schematic diagram of the two-phase flow loop. The experiments have been performed in a vertical air-water flow facility at Thermal-hydraulics and Reactor Safety Laboratory at Purdue University. The experimental facility is designed for simulating adiabatic vertical air-water two-phase flows under both upward and downward flow conditions. The test section consists of an acrylic round pipe with diameter (D) of $25.4 \mathrm{~mm}$ or $50.8 \mathrm{~mm}$, and height $(\mathrm{H})$ of $3.8 \mathrm{~m}$. The acrylic pipe is transparent, which provides a clear view of the flow for the entire length of the flow channel and helps to identify the flow regimes by visual inspection. The liquid flow rate is adjusted by valves and measured by an electro-magnetic flow meter with an accuracy of $3 \%$. Air is supplied by an air compressor with a maximum pressure of 60 bar. The air flow rate is adjusted by valves and measured by rotameters with an accuracy of $8 \%$. A sparger with a porous tip with 10 micron pores is employed as a bubble generator. The present injection unit produces almost uniform bubbles of approximately 1-2 $\mathrm{mm}$ in diameter at the test section inlet. More technical details about this facility can be found in Goda et al. (2004).

\subsection{Impedance meter}

An impedance meter is used to perform area averaged void fraction measurements with a sampling rate of $1 \mathrm{kHz}$ and an acquisition time of $60 \mathrm{~s}$. Two diagonal stainless steel electrodes are flush-mounted at the insulated wall in the measuring axial position. The electrodes are rectangle-shaped with round corners, a span- angle of $120^{\circ}$ and a height of $5 \mathrm{~mm}$. Delrin, a good electrical insulator, was used as a liner. An alternating current is supplied to the electrodes and the electrodes are connected to the electronic circuit, which is specially designed so that the output voltage of the circuit is proportional to the measured admittance, which is the inverse of impedance, between the electrodes. The measured admittance is normalized by the following equation:

$$
G_{n}=\frac{G_{m}-G_{1}}{G_{0}-G_{1}}
$$


where, $G_{m}$ is the instantaneous two-phase mixture admittance, $G_{0}$ is the admittance when void fraction is zero (i.e. single phase water) and $G_{1}$ is the impedance when the void fraction is unity (i.e. single phase air). Finally, the area averaged void fraction can be obtained from the measured admittance. Cross-calibration made with gamma densitometer shows that the void fraction is almost a linear function of the normalized admittance (Mi et al., 2001b). In addition, to convert from the measured admittance to the area-averaged void fraction value a calibration is made using differential pressure measurements at low liquid velocities (Mi et al., 2001a). Thus, the void fraction measured by the void meter is an instantaneous, area averaged void fraction. The uncertainty of the impedance meter can be estimated as $3 \%$ and the least counts of $1 \%$ (void fraction).

Once the signal from the impedance meter is converted into void fraction, the CPDF is obtained by sorting and normalizing the void fraction information. Figure 3 shows an example of set of CPDFs that corresponds to a constant liquid velocity and different gas velocity values. The CPDFs are grouped in flow regimes following the visual information. The shape of the CPDF depends on the interfacial distribution, i.e. flow regime, in the measured flow channel section (Mi et al., 1998; 2001b).

\subsection{Double sensor conductivity probes}

Conductivity probes are often used to measure various two-phase flow parameters, such as void fraction, interfacial velocity and interfacial area concentration (Kataoka et al., 1986; Hibiki et al., 1998; Kim et al., 2000). A double-sensor conductivity probe is installed at the center of the pipe and at the $3.4 \mathrm{~m}$ axial location after the test section inlet to measure the bubble chord length distributions for each flow condition. The optimum acquisition frequency and time length for obtaining the local bubble chord length entropy depends on the flow conditions (Julia et al., 2008). In this work, data were acquired for each flow condition with a frequency of $12 \mathrm{KHz}$ for 60 seconds using a 12 bit $\mathrm{A} / \mathrm{D}$ card. Previous works assure a correct CPDF calculation using these two acquisition parameters in the present flow conditions (Julia et al., 2008; Hernandez et al., 2006).

The first step in the CPDF calculation from conductivity probe signals consists of a signal pre-processing, where the noise in the raw signals is removed using a median filter. Then, the signals are normalized in relation to the maximum and minimum voltage values in the raw signal and are converted into step-signals using a threshold technique. Once the raw signals are pre-processed, the bubble residence time information is obtained from the front sensor signal. The last step consists of calculating, sorting and normalizing the bubble chord length. In order to transform the residence time information into the bubble chord length distribution it is necessary to calculate the interfacial velocity of each interface but, this is a time-consuming process. Instead, the interfacial velocity obtained from the cross-correlation between the two sensors has been used for the bubble chord length calculation. For the cross-correlation velocity calculation, the pre- 
processed signals are converted into step-signals using a threshold technique and the cross-correlation is computed. Since the distance between the sensors is known, it is straightforward to calculate the cross-correlation interfacial velocity. Different researchers have studied the accuracy of the cross-correlation interfacial velocity measured with conductivity (Kendoush et al., 1980; Julia et al., 2008) or hot film anemometry sensors (Gurau et al., 2004) in different flow regimes. These studies have shown that the crosscorrelation velocity can be used as a good estimator of the interfacial velocity. If a constant superficial liquid velocity is considered and the superficial gas velocity is increased from bubbly to churn-turbulent flow, the averaged difference between the cross-correlation and averaged interfacial velocities is lower than $15 \%$. The maximum error is in slug flow and Group 2 bubbles. However, the maximum error in the entropy calculation due to the use of the cross-correlation velocity is below $10 \%$ and does not affect to the identification process. Additionally, the computation time of the crosscorrelation velocity is two orders of magnitude lower than the interfacial velocity. This fact is especially important in fast identification process and justifies the use of the crosscorrelation velocity. Figure 4 shows an example of a set of bubble chord length CPDFs taken in the center of a pipe. The CPDFs are grouped in flow regimes following the visual information.

\subsection{Probabilistic neural network}

The human brain inspired the development of the ANN and their initial application to practical problems started in the 1950s. Numerous ANN applications have been developed, mainly solving problems where input and output values are known, but the translation into a mathematical equation is difficult. A Probabilistic Neural Network (PNN) is an ANN used for data classification tasks. In the current study, a PNN was chosen to solve the problem of classifying the calculated flow regime indicators (inputs) into the five different flow regimes (classes).

PNNs belong to the family of the radial basis ANN, feature a feed-forward architecture and a fast supervised training algorithm, as is usually performed in a single pass. An example of a PNN structure or architecture is shown in Figure 5. The PNN has four layers. The input layer contains as many elements as parameters required to describe the test cases to be classified. In this study, the number of parameters would range between one and ten input components, and in Figure 5, two parameters are considered. The next layer is the hidden layer, with as many elements as test cases in the training data set. In the architecture shown in Figure 5, only seven test cases are depicted. The third layer is the pattern or summation layer, which has as many elements are there are classes to be recognized: three in the example of Figure 5 or five in the problem of flow regime classification. The final layer is the decision or output layer, which assigns the final classification of each pattern to the largest probability within the different classes.

The PNN model is first taught with a training data set, which in this study consists of 
flow regime indicators along with their correct classification. PNN are based on the combination of the Bayes decision strategy and Parzen's method of PDF approximation (Specht 1988; Specht 1990; Parzen 1962).The classification decision is made by means of the PDF of each flow regime calculated using the training patterns. The goal of training is to approximate the PDF of the underlying distribution of the training pattern. Finding the PDF of the training patterns is based on its approximation by superposition of a certain function, which in this study is the Gaussian function. When applying a PNN it is important to ensure a proper selection of the width parameter in the curve, which controls how spread the Gaussian curve is. The spread parameter ranges between 0 and 1 and the smaller the spread value is, the more selective the corresponding neuron is and, in turn, the more the ANN acts as a nearer neighbour classifier. A trial and error method was used to find the optimum spread value. Once the training process is finished, the PNN classifies the new test cases based on the PDF generated with the training data.

The neural networks were trained, using a random set of $90 \%$ of the available data and predicting the remaining $10 \%$. The process was repeated 50 times for each ANN configuration, which resulted in 50 different ANNs. These 50 trained ANNs were assembled in an ANN committee, in order to compensate for the differences in the individual networks. The final classification results were obtained by averaging the randomly resulting predictions provided by all the ANNs that integrate the ANN committee. More technical information about the neural networks used in this work can be found in Hernández et al. (2006).

\subsection{Area-averaged void fraction and local bubble chord length entropies definition}

The entropy computation is based on the CPDF distribution of the area-averaged void fraction or local bubble chord length. The area-averaged void fraction entropy $\left(S_{a}\right)$ and the bubble chord length entropy $\left(S_{B}\right)$ are defined as follows,

$\mathrm{S}_{\alpha \text { or } \mathrm{B}}=\frac{1}{\mathrm{~N}} \sum_{\mathrm{i}=0}^{\mathrm{i}=\mathrm{N}}-\mathrm{p}_{\mathrm{i}} \ln \left(\mathrm{p}_{\mathrm{i}}\right)$

where $p_{i}$ is the cumulative probability of finding a $i_{t h}$ - area-averaged void fraction or bubble chord length value in the CPDF and $\mathrm{N}$ is the number of samples.

The relation between the flow regime, conductivity probe signals and $S_{B}$ value is analyzed in Figure 6 . This figure is interpreted from left to right. The first column provides examples of images of the typical five flow regimes considered in this study $(B, C B, C S$, $\mathrm{CT}$ and $\mathrm{A}$ ). The second column of Figure 6 shows examples of typical conductivity probe signals time series for every flow regime. The CPDF of the signals are also plotted in the third column of the figure. The upper limit of the bubble chord length CPDF is 2 meters. The CPDF for the bubbly flow extends to $1 \mathrm{~cm}$ with a constant slope. The $S_{B}$ value that corresponds to bubbly flow (forth column of the figure) is low since most of the CPDF 
values are equal to one.

In the case of the cap-bubbly, slug and churn-turbulent CPDFs it is possible to distinguish two different regions, corresponding to the two different types of bubbles found in these regimes. In the three cases, the regions corresponding to the small bubbles are very similar to the CPDF of the bubbly flow, but the larger bubbles contribution zone has different slopes. In the case of cap-bubbly flow, the contribution of the small bubbles reaches to the $40 \%$ of the CPDF, and the cap bubbles contribution extends to $10 \mathrm{~cm}$. In the case of slug flow, the small bubbles contribution reaches to the $30 \%$ of the CPDF, and the Taylor bubbles contribution extends to $60 \mathrm{~cm}$, with a gap between $1 \mathrm{~cm}$ and $10 \mathrm{~cm}$. In the case of churn-turbulent flow, the contribution of the small bubbles reaches to the $20 \%$ of the CPDF, and the churn-turbulent bubbles contribution extends to $2 \mathrm{~m}$. The gap in the bubble chord lengths found in the slug flow disappears due to the break-up of the Taylor bubbles. The $S_{B}$ values that correspond to these flow regimes show a continuous increment due to the CPDF function enlargement towards longer bubble chord lengths and its form.

In the case of annular flow, with extremely long Taylor bubbles, the CPDF should be zero in all the range in the center of the flow channel. However, the figure shows a slight increment for bubble chord lengths larger than $1 \mathrm{~m}$. These measurements correspond to intermittent liquid bridges that are present in the experimental loop, which were identified as long churn-turbulent bubbles. The $S_{B}$ value drops to values closer to those found in bubbly flow.

In a similar way, Figure 7 analyzes the relation between the flow regime, impedance meter signal and $S_{\alpha}$ value. The information provided by the $S_{\alpha}$ value for the bubbly, cap-bubbly, slug and churn-turbulent flow regimes is similar to the one provided by $S_{B}$. The main changes between $S_{\alpha}$ and $S_{B}$ are found in the annular flow regime. The $S_{\alpha}$ value does not drop in this flow regime due to the fact that the $\alpha$ values are areaaveraged. Then, the variations of thickness in the liquid film have a strong effect in the impedance meter signal and the CPDF presents a similar shape to the one found for churn-turbulent flow regime.

\section{Results and Discussion}

In this work, both area-averaged void fraction and bubble chord length entropies have been calculated for 242 flow conditions in vertical upward two-phase flows in $25.4 \mathrm{~mm}$ and $50.8 \mathrm{~mm}$ pipes. The superficial gas and liquid velocity conditions are $0.13 \mathrm{~m} / \mathrm{s}<j_{\mathrm{f}}<5$ $\mathrm{m} / \mathrm{s}$ and $0.01 \mathrm{~m} / \mathrm{s}<j_{\mathrm{g}}<25 \mathrm{~m} / \mathrm{s}$ respectively. Figure 8 shows the flow regime maps by visual observation for both configurations. 


\subsection{Area-averaged void fraction entropy}

Figure 9 shows the evolution of the area-averaged void fraction entropy for upward flow in a $25.4 \mathrm{~mm}$ diameter pipe at the axial location of $z / D=144$ and several superficial liquid velocity values. The flow regime information has been obtained from the visual flow regime map of Figure 8a) and they are shown only for indicative purposes and for low superficial liquid velocities.

It is observed that the entropy provides quantitative information about the disorder in the area-averaged void fraction distribution as analyzed in Figures 6 and 7 . It has a low value for bubbly flows. An increment in the gas flow rate increases the entropy as we find a wider distribution of bubble types in the cap-bubbly, slug and churn-turbulent flow regimes. The maximum occurs in the churn-turbulent flow regime and it is constant in annular flow. The entropy values found for the annular flow regime does not drop because $S_{\alpha}$ is an area-averaged quantity and the entropy contribution of the two-phase flow near the walls in this flow regime is quite important. In addition, the entropy values show a monotonic increment with the superficial gas velocity and, for the same flow regime, do not depend on the superficial liquid velocity. Some discontinuities in the entropy values can be found in the flow regime transition zones. These discontinuities do not affect the flow regime identification process. This fact can be related with the experimental uncertainty in the CPDF calculation. However, more experimental data is needed in order to analyze it in detail. This fact makes the mean $S_{a}$ a good flow regime indicator aspirant in upward flow conditions. The evolution of the $S_{a}$ values with the superficial gas and liquid velocities in a $50.8 \mathrm{~mm} D$ pipe is almost identical to the one shown in Figure 9, confirming that the pipe diameter does not play an important role in the flow regime transitions in upward flow conditions (Taitel et al., 1980; Mishima \& Ishii; 1984)

\subsection{Local bubble chord length entropy}

Figure 10 shows the $S_{B}$ calculated in the center of the pipe for two constant liquid velocity conditions $\left(\mathrm{j}_{\mathrm{f}}=0.03 \mathrm{~m} / \mathrm{s}\right.$ and $\left.\mathrm{j}_{\mathrm{f}}=1.27 \mathrm{~m} / \mathrm{s}\right)$, superficial gas velocities ranging from 0 to $10 \mathrm{~m} / \mathrm{s}$ and at the location of $z / D=65$.

It is observed that the $S_{B}$ dependence with the superficial gas velocity in the center of the pipe is quite similar to the one found for $S_{a}$, except for the annular flow. In this way, the $S_{B}$ values are very low for low gas velocity conditions (bubbly flow) and increase with the gas velocity (cap-bubbly, and slug flow). When the gas velocity is between $1 \mathrm{~m} / \mathrm{s}$ and $4 \mathrm{~m} / \mathrm{s}$ the $S_{B}$ is almost constant (churn-turbulent flow). For gas velocity higher than $5 \mathrm{~m} / \mathrm{s}$ the $S_{B}$ drops (annular flow). This last fact is because the bubble chord length entropy is a local measurement, so measurements in the gas core of the annular flow are possible represented by very long chord lengths. 
From the above, it is clear that the bubble chord length entropy presents better characteristics than the area-averaged void fraction entropy as flow regime indicator. Its main advantages are the higher dependency of the entropy value with the flow regime (the ratio between the entropy values of $\mathrm{CT}$ and $\mathrm{B}$ flow regimes is much higher in the case of $S_{B}$ ), the drop of the entropy in the annular flow and the possibility of using several entropy values in different radial locations since it is a local measurement (the later advantage is not studied in the present work).

\subsection{Flow regime identification results}

In this section, the area-averaged void fraction and bubble chord length entropy capabilities as flow regime indicators will be discussed. Thus, the flow regime classification results obtained by both entropies have been compared to those obtained by other statistical parameters using different durations of the input signals.

\subsubsection{Area-averaged void fraction entropy}

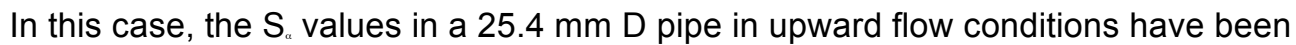
used as inputs of a PNN. The obtained results have been compared with those provided by the same PNN, but using other statistical parameters of the void fraction CPDF distribution. The statistical parameters used are as follows:

- Mean value and standard deviation of the PDF (Mean+std): this set of statistical parameters is the most used in the flow regime identification works. The PDF is calculated by differentiation of the CPDF and the mean and standard deviation are calculated in the usual form (Mi et al. 1998; 2001b).

- $\quad$ Principal component analysis of the CPDF (PCA): it is a useful statistical technique that compresses the data by reducing the number of dimensions without much loss of information. This is done by transforming the data into orthogonal components, so that a set of possible correlated variables are reduced into a smaller set of uncorrelated variables. Components which contribute less than a specified fraction of the total variation of the input data are eliminated, and only the reduced number of variables which best explain the variance of the data are preserved. Following this methodology, the CPDF vectors were reduced up to six components with a variance contribution higher than $0.1 \%$. More details about this methodology can be found in Mohamad-Saleh and Hoyle (2002) and Hernandez et al. (2006).

- Four indices of the CPDF (4IND): this is a simple and fast procedure to characterize the CPDF vectors. In this case the four indices of the vectors where CPDF values of $0.25,0.5,0.75$ and 1 were reached are selected as being characteristic of the CPDF shape. Afterwards, these four values are normalized so that they fall within the range $[-1,1]$. More details about this methodology can be found in Hernandez et al. 
The flow regime identification results have been compared to those given by visual information in Figure 8 a). Table 1 provides the identification results for different CPDF processing times, with input signal durations ranging from 0.1 to 30 seconds.

The second row in Table 1 indicates the number of components employed for each indicator, varying from one in the case of the area-averaged void fraction entropy up to ten in the case of PCA. It is important to remark that for different CPDF processing times the PCA of the input vector have been recalculated, and therefore different number components are obtained for each case.

Table 1 shows the final prediction of the ANN committee for each of the 20 combinations of indicators and processing times. The percentage indicates the agreement between visual classification and the ANN committee final classification for each of the 109 test cases for these conditions. For a range of times between 0.1 up to 30 seconds the predicting quality of the ANN is maintained for the four different indicators.

PCA, $S_{a}$ and 4IND indicators provide similar agreements with the visual information, always above those results obtained with the other indicator. The results for these three indicators present most of the time total agreement with visual information. The decrease of processing time up to 0.1 seconds in the signal used to calculate the inputs do not have a relevant role in the quality of the ANN classification results. The best classification results with the least provided information are obtained for the area-averaged void fraction entropy, with only one component required for the identification. This means that the area-average entropy is able to compact the flow regime information more efficiently than the other statistical parameters. This fact makes the area-averaged void fraction entropy suitable for fast identification processes since the time needed for the ANN training and identification process is dependent on the amount of information used in the process. As an example, when comparing entropy as a single input instead of the eight inputs for the PCA case for the CPDF processing time of 1 second, a reduction of $15 \%$ in the PNN training time is observed. In addition, the time needed for the entropy calculation is much lower than that used for the PCA parameters, within the range of one order of magnitude.

It is interesting to study the test conditions with higher misclassified predictions. Figure 11 provides the flow regime maps obtained for every flow regime indicator and for a processing time of 1 second. Figure 10 shows, for the different indicators and always for CPDF processing time of 1 second, the percentage of failure PNN predictions for the different test cases. If one test case presents percentage of failure lower than $50 \%$, the PNN classification agrees with the visual one. On the contrary, if this value is higher than $50 \%$, the corresponding test case has been misclassified by the ANN commitee. It is observed that the flow conditions with certain percentage of identification failure are quite similar for PCA, 4IND and $S_{a}$ indicators, mainly concentrated in the $S$ to $C T$ flow regime transition zone. The mean-std indicator presents problems with all the flow conditions 
except for the B and CT flow regimes.

If input signal durations lower than 0.1 seconds are processed, the ANN predictions worsen for all the parameters. This duration agrees with the time scale given by the bubble characteristic length (Laplace length) divided by the average bubble interfacial velocity and modulated by the void fraction in bubbly flows.

\subsubsection{Bubble chord length entropy}

In this case, the $S_{B}$ values in a $50.8 \mathrm{~mm} D$ pipe in upward flow conditions have been used as inputs for the ANN. The set of CPDF that correspond to the probe located at the center of the pipe has been used.

The four flow regime indicators described in the previous section have been also used and the flow regime identification results have been compared to those given by visual information in Figure 8b). Table 2 provides similar information as that Table 1 but now for the case of bubble chord length indicators. As in Table 1, the prediction for $S_{B}, P C A$ and 4IND for processing times in the range of 1 to 30 seconds are quite similar among them. Again, the mean+std indicator presents the lowest classification results. No much influence of the CPDF processing time of the bubble chord length is neither observed for this range. The best identification results with the lowest required components are obtained for $S_{B}$ indicator, with PNN training time reductions similar as those presented for the case of $S_{\text {. }}$. However, ANN predictions worsen when processing time diminishes to 0.1 seconds, as the quality of the CPDF decreases and therefore the representativeness of the indicators for the corresponding flow regime is lower. However, the results provided by the impedance meter measurements based parameters where accurate for this input signal duration. This last fact is easily explained since the conductivity probe and the impedance meters are a local and an area-average measurement, respectively. Thus, the probability of detecting a bubble with the conductivity probe is lower than with the impedance meter if short measurement periods are considered.

Figure 13 shows the same information as that in Figure 10, but using the conductivity signal from the probe at the center of the pipe. The mean+std indicator presents identification problems spread over all the flow regimes. For the other indicators, the identification failures are concentrated in bubbly flow and churn-turbulent flow conditions.

Finally, it was shown in Sections 3.1 and 3.2 that the $S_{B}$ have better characteristics as flow regime indicator than $S_{\text {. }}$. However, the results provided by both entropies are quite similar if input signals duration longer than 0.1 seconds are considered and the failures in the flow regime identifications are located in the same flow regime map zones.

\section{Conclusions}

The area-averaged void fraction and bubble chord length entropies have been 
introduced as flow regime indicators in two-phase flow systems. The entropy provides quantitative information about the disorder in the area-averaged void fraction or bubble chord length distributions. The area-averaged void fraction entropy is based on impedance meter signals and the bubble chord length entropy in conductivity probe signals. Both entropies have been calculated for 242 flow conditions in upward flow in pipes.

The physical meaning of both parameters has been analysed using the visual flow regime map information. In this way, it is possible to observe a clear dependence of the entropy value with the flow regime in upward flows. Both area-averaged void fraction and bubble chord length entropies present suitable characteristics as flow regime indicators.

The area-averaged void fraction and the bubble chord length entropies capability as flow regime indicators has been checked with other statistical parameters of the void fraction and bubble chord length CPDF distributions (Mean+std, 4IND and PCA). Additionally, the variation of the indicators classification capability with different CPDF processed with different input signals durations has been evaluated.

Classification efficiency using these entropy variables as inputs for the ANN commitee are similar to those obtained by PCA or 4IND methods ( $99 \%$ efficiency for areaaveraged entropy and $\sim 96 \%$ efficiency for bubble chord length entropy). However, the entropy is capable to reduce the relevant information of the flow regimes in only one significant and useful variable. In addition, the computation time for the entropy is much lower than for the PCA. In terms of generating any empirical model to predict flow regimes, reducing the independent variables to only one decreases the required experimental data. Additionally for the case of using neural networks using one variable as input represents less training time and faster predictions.

The time needed for obtaining good classification results depends on the type of signal used. If area-averaged void fraction signals obtained from an impedance meter are used a duration of 0.1 seconds is enough for obtaining accurate classifications (almost $100 \%$ agreement with visual information). If conductivity probe signals are used (bubble chord length distributions) a temporal length of 1 second is necessary to accurate classifications to be obtained ( $96 \%$ agreement with visual information). This difference in the minimum temporal length requirement can be explained since conductivity probes provide local measurements that are more dependent on the input temporal length.

\section{Acknowledgements}

This work was performed at Purdue University under the auspices of Mitsubishi Heavy Industries $L t d$. through the Institute of Thermal-hydraulics. 


\title{
Nomenclature
}

\author{
Abbreviations \\ A annular flow regime \\ ANN artificial neural network \\ B bubbly flow regime \\ CB cap-bubbly flow regime \\ CT churn-turbulent flow regime \\ CPDF cumulative probability distribution function \\ GFR global flow regime \\ IND indices \\ LFR local flow regime \\ PCA principal components analysis \\ PDF probability distribution function \\ PNN probabilistic neural network \\ PSD power spectral density \\ S slug flow regime \\ SONN self-organizing neural network \\ $\mathrm{z} \quad$ axial location $(\mathrm{m})$
}

\section{Symbols}

D pipe inner diameter (m)

G admittance (-)

$\mathrm{H} \quad$ pipe height $(\mathrm{m})$

j superficial velocity $(\mathrm{m} / \mathrm{s})$

L Chord length

$\mathrm{N}$ number of samples

$\mathrm{p} \quad$ cumulative probability

S entropy (-)

$\mathrm{z} \quad$ axial location $(\mathrm{m})$ 


\section{Greek letters}

$\alpha \quad$ void fraction

\section{Subscripts}

0 zero void fraction

1 unity void fraction

$\alpha \quad$ void fraction

g gas

$\mathrm{f} \quad$ liquid

m mixture

n normalized

B bubble chord length 


\section{References}

Cai, S., Toral, H., Qiu, J. and Archer J. S., (1994). Neural network based objective flow regime identification in air-water two-phase flow. Can. J. Chem. Eng. 72, 440-445.

Elperin, T and Klochko, M. (2002). Flow regime identification in a two-phase flow using wavelet transform. Exp. in Fluids 32, 674-682.

Goda, H., Hibiki, T., Kim, S., Ishii, M. and Uhle, J. (2004). Drift flux model for downward two-phase flow. Int. J. Heat Mass Transfer 47, 4101-4118.

Gurau, B., Vasallo, P. and Keller, K. (2004). Measurement of gas and liquid velocities on an air-water two-phase flow using cross-correlation of signals from a double sensor hotfilm probe. Exp. Thermal Fluid Science 28, 495-504.

Hernandez, L., Julia, J. E., Chiva, S., Paranjape, S., Ishii, M. (2006). Fast classification of two-phase flow regimes based on conductivity signals and artificial neural networks.

Meas. Sci. Technol. 17, 1511-1521.

Hibiki, T., Hogsett, S., Ishii, M. (1998). Local measurement of interfacial area, interfacial velocity and liquid turbulence in two-phase flow. Nuclear Eng. Des. 184, 287-304.

Ishii, M. and Mishima, K. (1984). Two-fluid model and hydrodynamic constitutive relations. Nucl. Eng. Des. 82,107-126.

Ishii, M. and Hibiki, T. (2007). Thermo-fluid Dynamics of Two-Phase Flow. Springer. Jones, O. C. and Zuber, N. (1975). The interrelation between void fraction fluctuations and flow patterns in two-phase flow. Int. J. Multiphase Flow 3, 273-306.

Julia, J. E., Liu, Y., Paranjape, S. and Ishii, M. (2008). Local flow regimes analysis in vertical upward two-phase flow. Nuclear Engineering Design 238, 156-169.

Kataoka, I., Ishii, M. and Serizawa, A. (1986). Local formulation and measurements of interfacial area concentration in two-phase flow. Int. J. Multiphase Flow 12, 505-529.

Kendoush, A. A., Zaki, R. Y. and Shakir, B. M. (1980). Slip measurement in downward two-phase bubbly flow using cross-correlation technique. Multiphase Transport:

Fundamentals, Reactor Safety, Applications Vol.5, T. Nejat Veziroglu Ed. Hemisphere Publishing Corporation.

Kim, S., Fu, X. Y., Wang, X. and Ishii, M. (2000). Development of the miniaturized foursensor conductivity probe and the signal processing scheme. Int. J. Heat Mass Transfer 43, 4101-4118.

Lee, J. Y., Ishii, M. and Kim, N. S. (2008a). Instantaneous and objective flow regime identification method for the vertical upward and downward co-current two-phase flow. International Journal of Heat and Mass Transfer 51, 3442-3459. 
Lee, J. Y., Kim, N. S., and Ishii, M. (2008b). Flow regime identification using chaotic characteristics of two-phase flow. Nuclear Engineering and Design, 238, 945-957.

Matsui, G. (1984). Identification of flow regimes in vertical gas-liquid two-phase flow using differential pressure fluctuations. Int. J. Multiphase Flow 10, 711-720.

Mi, Y., Ishii, M. and Tsoukalas, L. H. (1998). Vertical two-phase flow identification using advanced instrumentation and neural networks. Nucl. Eng. Des. 184, 409-420.

Mi, Y., Ishii, M. and Tsoukalas, L. H. (2001a). Investigation of vertical slug flow with advanced two-phase. Nucl. Eng. Des. 204, 69-85.

Mi, Y., Ishii, M. and Tsoukalas, L. H. (2001b). Flow regime identification methodology with neural networks and two-phase flow models. Nucl. Eng. Des. 204, 87-100.

Mishima, K. and Ishii, M. (1984). Flow regime transition criteria for upward two-phase flow in vertical tubes. Int. J. Heat Mass Transfer 27, 723-737.

Mohamad-Saleh, J. and Hoyle, B. S. (2002). Determination of multi-component flow process parameters based on electrical capacitance tomography data using artificial neural networks. Meas. Sci. Technol. 13, 1815-1821.

Parzen E. (1962). On estimation of a probability density function and mode Annals of Mathematical Statisics 3, 1065-76.

Ruzicka, M. C., Drahos, J. Zahradnik, J. And Thomas, N. H. (1997). Intermittent transition from bubbling to jetting regime in gas-liquid two-phase flow. Int. J. Multiphase Flow 23, 671-682.

Sharma, H., Das, G., Samanta, A.N., (2006). ANN-based prediction of two phase gasliquid flow patterns in a circular conduit. AIChE J. 52, 3018-3028.

Specht D F. (1988). Probabilistic neural networks for classification, mapping or associative memory Proc. of IEEE Int. Conf. on Neural Networks 1 527-30

Specht D F (1990). Probabilistic neural networks Neural Netw. 3 109-18.

Taitel, T., Barnea, D. and Dukler, A. E. (1980). Modelling flow pattern transitions for steady upward gas-liquid flow in vertical tubes. AIChE. J. 26, 345-354.

Tutu, N. K. (1982). Pressure fluctuations and flow pattern recognition in vertical two phase gas-liquid flows. Int. J. Multiphase Flow 8, 443-447.

Zhang, Z. and Shi, L. (1999). Shannon entropy characteristics in two-phase flow systems. J. Appl. Physics 85, 7544-7551. 


\section{Figure captions}

Figure 1. Two-phase flow patterns representation for upward flow, a) bubbly, b) capbubbly, c) slug, d) churn-turbulent and e) annular

Figure 2. Two-phase flow loop

Figure 3. CPDF distribution set of void fractions for upward flow, $25.4 \mathrm{~mm}$ diameter pipe, $\mathrm{j}_{\mathrm{f}}=1.25 \mathrm{~m} / \mathrm{s}$ and $0.01 \mathrm{~m} / \mathrm{s}<\mathrm{j}_{\mathrm{g}}<16 \mathrm{~m} / \mathrm{s}$

Figure 4. CPDF distribution set of bubble chord lengths for upward flow, $50.8 \mathrm{~mm}$ pipe, $\mathrm{j}_{\mathrm{f}}=1.27 \mathrm{~m} / \mathrm{s}$ and $0.01 \mathrm{~m} / \mathrm{s}<\mathrm{j}_{\mathrm{g}}<4 \mathrm{~m} / \mathrm{s}$

Figure 5. PNN architecture for two inputs and three classes

Figure 6. Correspondence between the flow regimes and $S_{B}$

Figure 7. Correspondence between the flow regimes and $S_{a}$

Figure 8. Visual flow regime map for upward flow a) upward flow $25.4 \mathrm{~mm} \mathrm{D}$ pipe and b) upward flow $50.8 \mathrm{~mm}$ D pipe

Figure 9. Evolution of the area-averaged void fraction entropy with the superficial gas and liquid velocities in a $25.4 \mathrm{~mm} \mathrm{D}$ pipe in upward flow conditions

Figure 10. Evolution of the bubble chord length entropy with the superficial gas velocity in a $50.8 \mathrm{~mm} \mathrm{D}$ pipe in upward flow conditions

Figure 11. Flow maps predicted with PNN with processing time of 1 second and using a) Mean+std, b) $S_{a}$, c) $4 I N D$ and d) PCA as flow regime indicators

Figure 12. Flow maps with the percentage of identification failures of the PNN with processing time of 1 second using impedance probe signals and a) Mean+std, b) $S_{a}$, c) 4IND and d) PCA as flow regime indicators

Figure 13. Flow maps with the percentage of identification failures of the PNN using conductivity probe signals and a) Mean+std, b) $S_{a}$, c) 4IND and d) PCA as flow regime indicators 


\section{Table captions}

Table 1. Flow regime identification results for area-averaged void fraction.

Table 2. Flow regime identification results for bubble chord length indicators. 


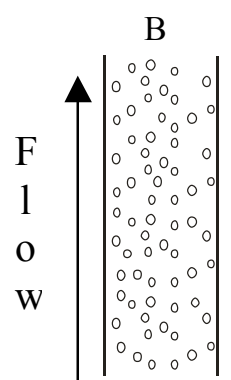

a)

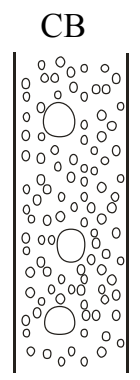

b)

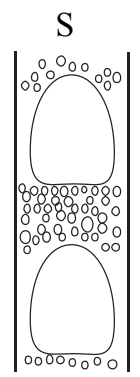

c)

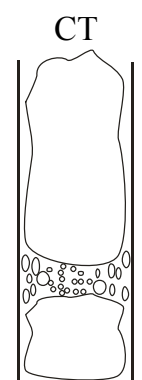

d)
A

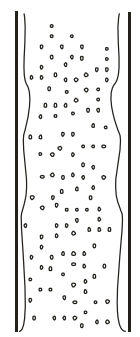

e)

Figure 1. Two-phase flow patterns representation for upward flow a) bubbly, b) capbubbly, c) slug, d) churn-turbulent and e) annular 


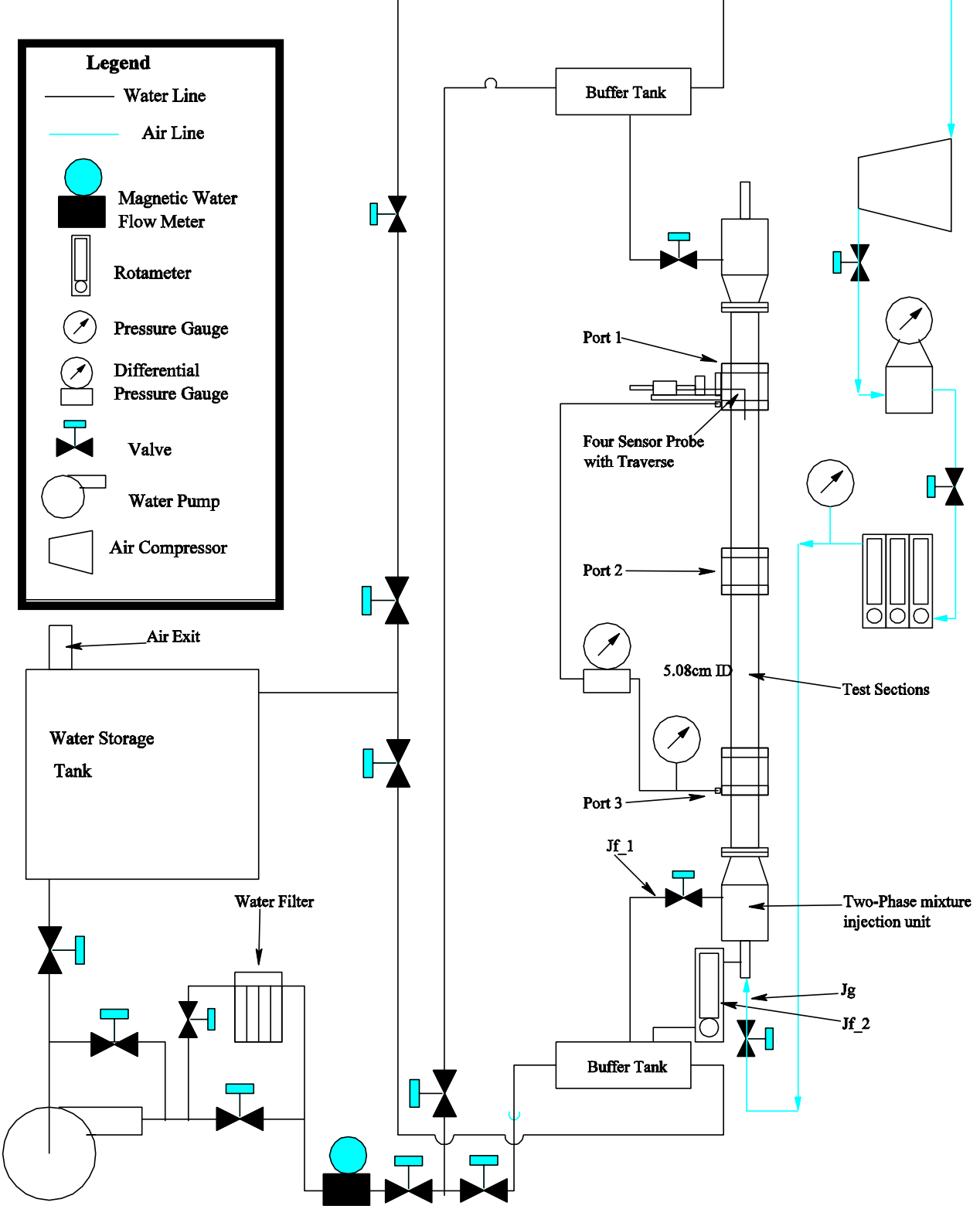

Figure 2. Two-phase flow loop 


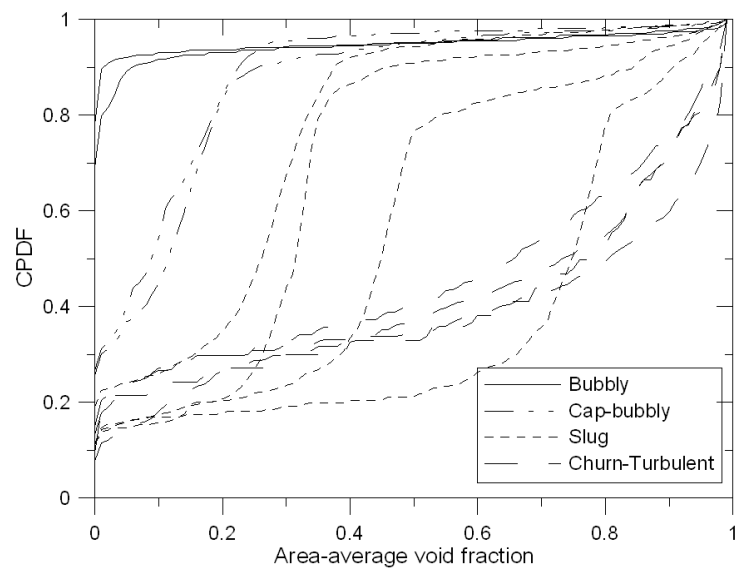

Figure 3. CPDF distribution set of void fractions for upward flow, $25.4 \mathrm{~mm}$ diameter pipe, $\mathrm{j}_{\mathrm{f}}=1.25 \mathrm{~m} / \mathrm{s}$ and $0.01 \mathrm{~m} / \mathrm{s}<\mathrm{j}_{\mathrm{g}}<16 \mathrm{~m} / \mathrm{s}$ 


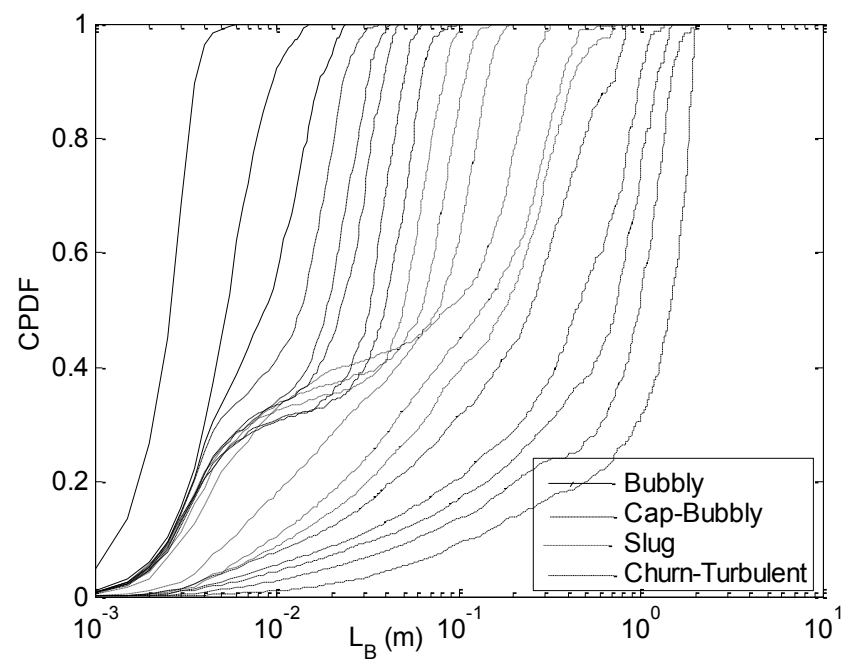

Figure 4. CPDF distribution set of bubble chord lengths for upward flow, $50.8 \mathrm{~mm}$ pipe, $\mathrm{j}_{\mathrm{f}}=1.27 \mathrm{~m} / \mathrm{s}$ and $0.01 \mathrm{~m} / \mathrm{s}<\mathrm{j}_{\mathrm{g}}<4 \mathrm{~m} / \mathrm{s}$. 


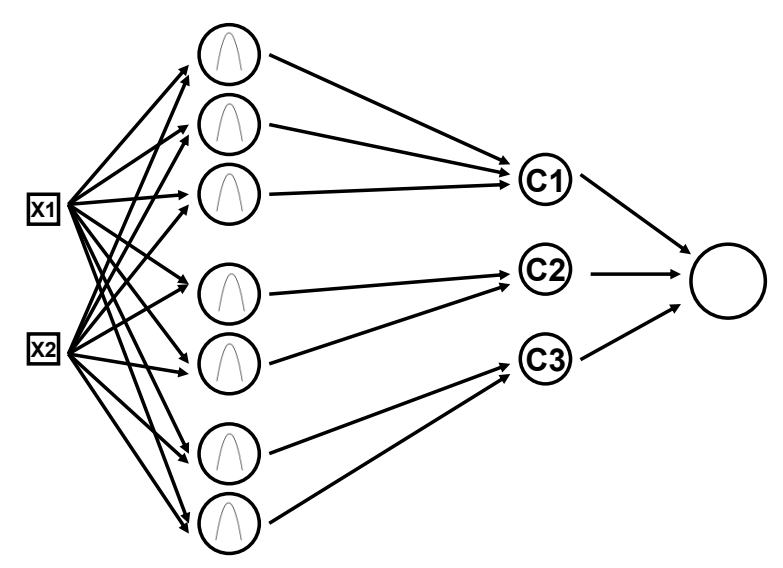

Figure 5. PNN architecture for two inputs and three classes 

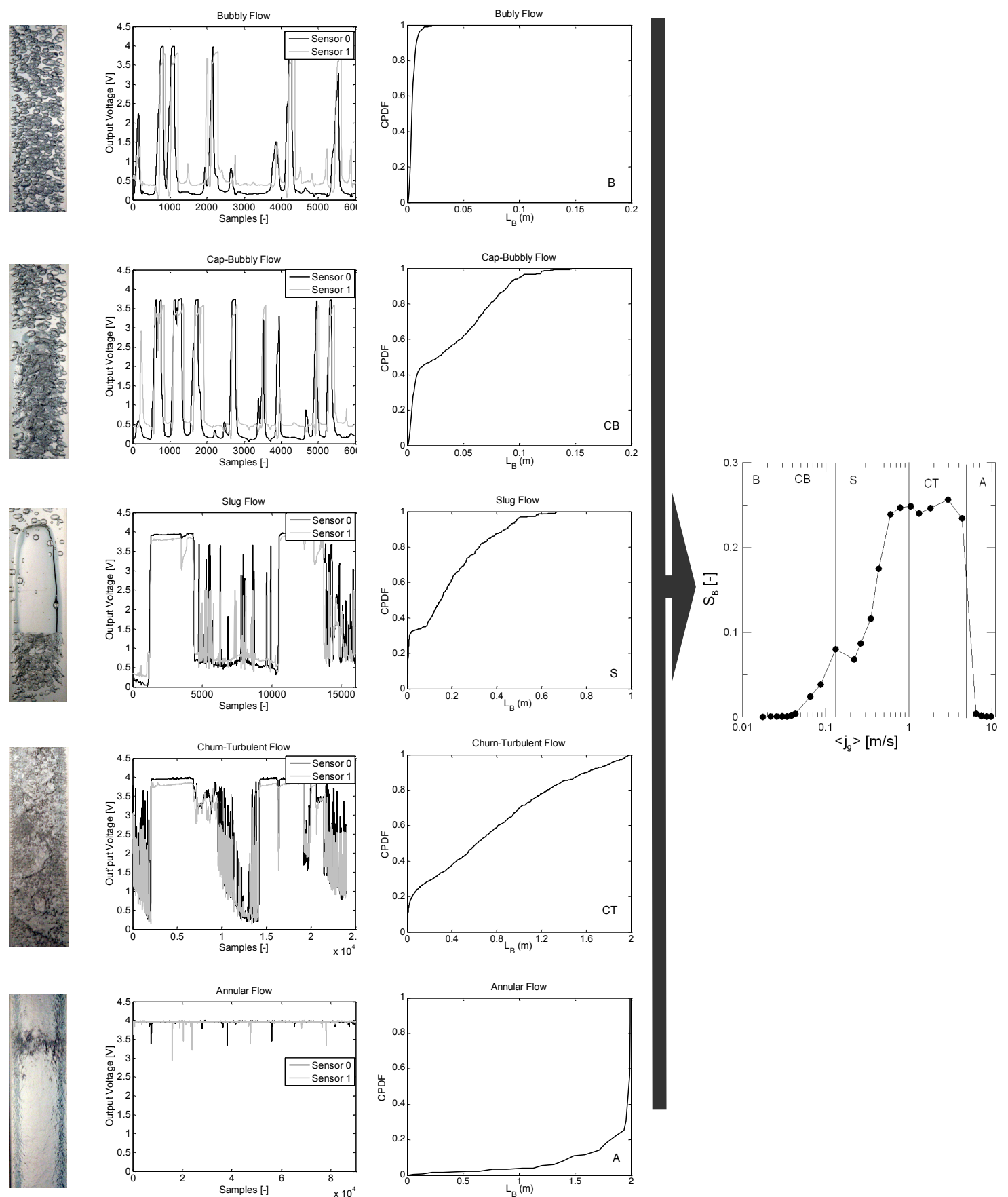

Figure 6. Correspondence between the flow regimes and $S_{B}$

Figure 6. 

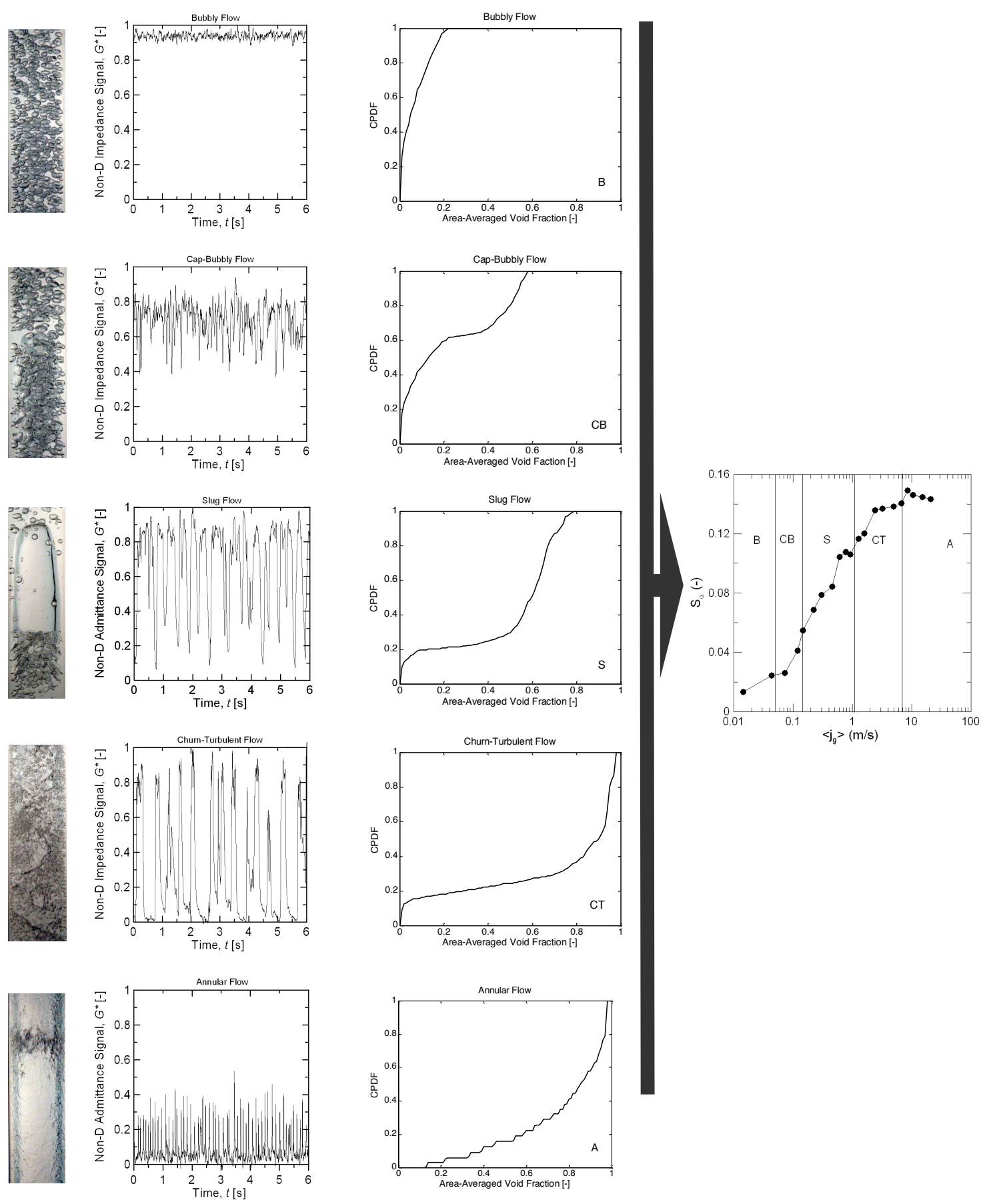

Figure 7. Correspondence between the flow regimes and $S_{a}$

Figure 7. 


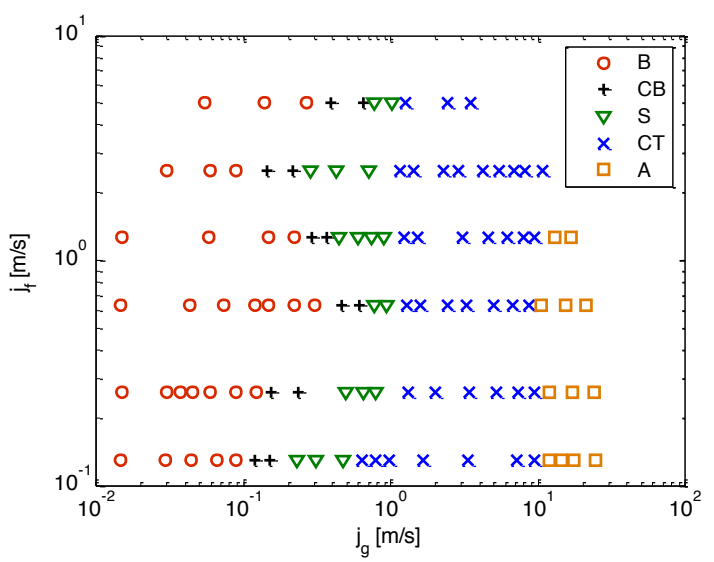

a)

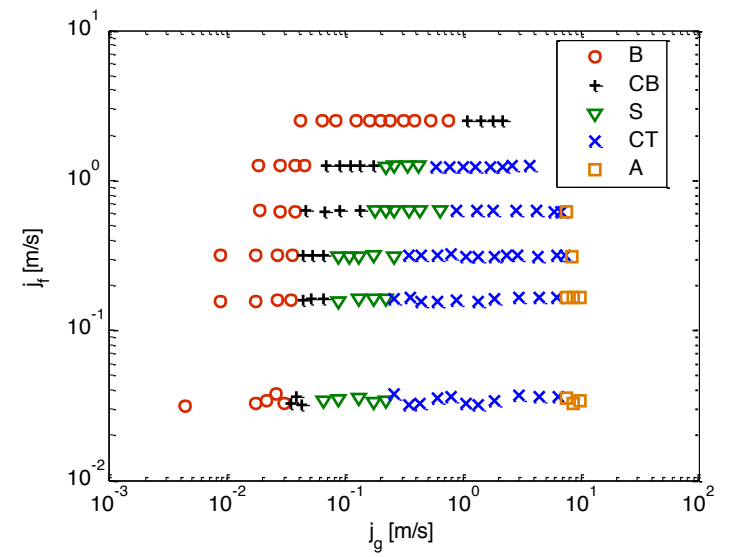

b)

Figure 8. Visual flow regime map for a) upward flow $25.4 \mathrm{~mm} \mathrm{D} \mathrm{pipe,} \mathrm{b)} \mathrm{upward} \mathrm{flow} 50.8$ $\mathrm{mm}$ D pipe 


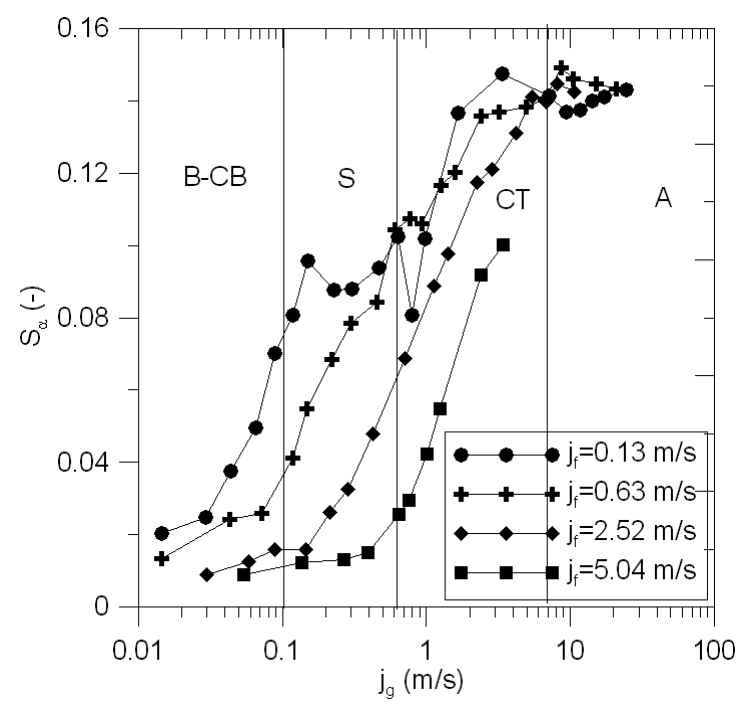

Figure 9. Evolution of the area-averaged void fraction entropy with the superficial gas and liquid velocities in a $25.4 \mathrm{~mm}$ D pipe in upward flow conditions 


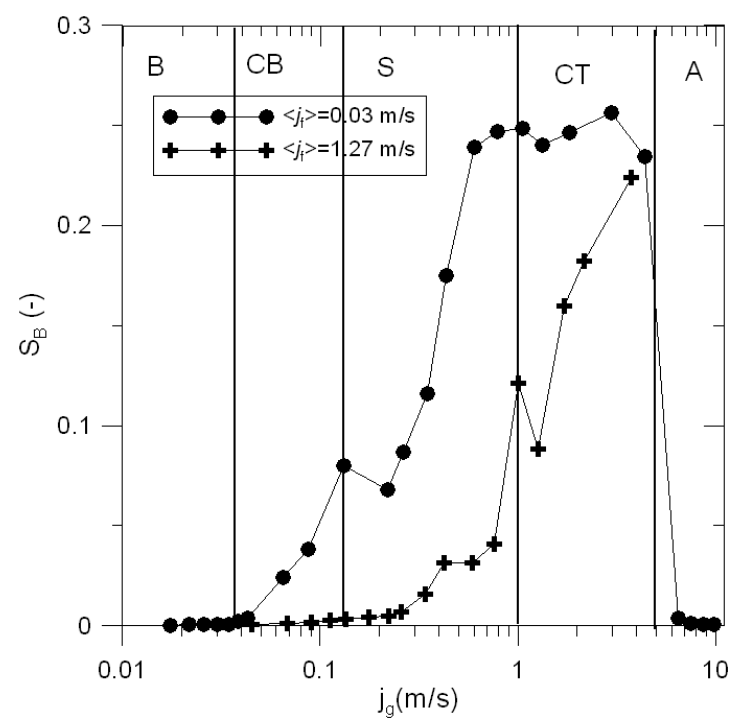

Figure 10. Evolution of the bubble chord length entropy with the superficial gas velocity in a $50.8 \mathrm{~mm} \mathrm{D}$ pipe in upward flow conditions 


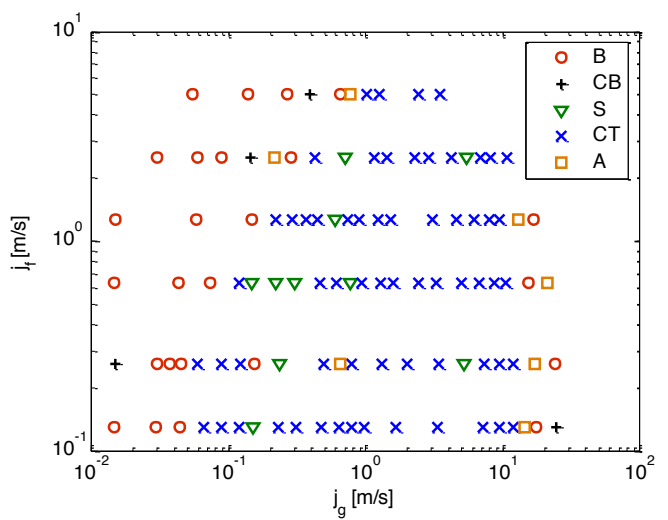

a)

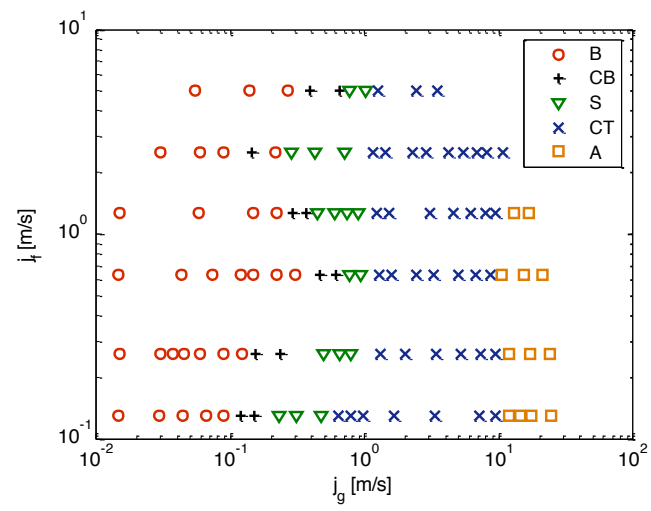

c)

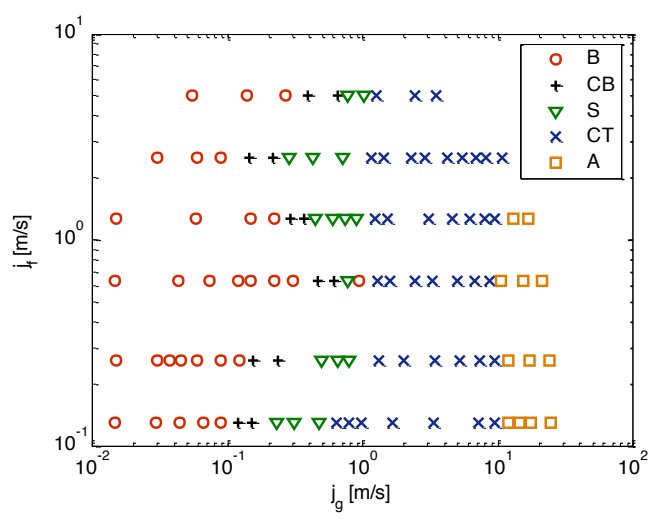

b)

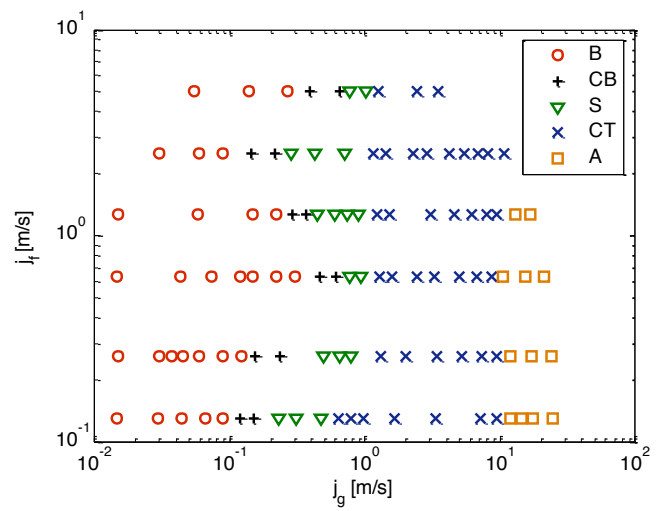

d)

Figure 11. Flow maps predicted with PNN with processing time of 1 second and using a) Mean+std, b) $S_{\text {a, c) }} 4$ IND and d) PCA as flow regime indicators 


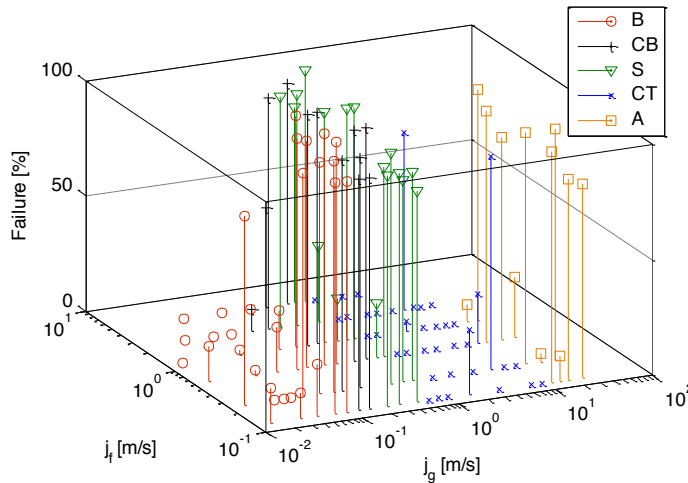

a)

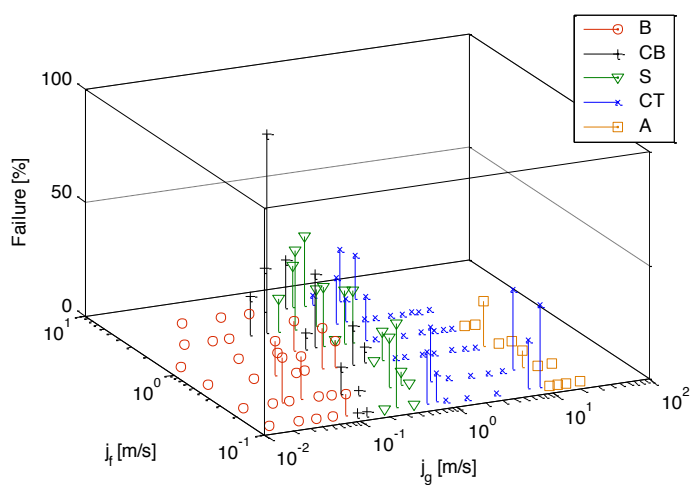

c)

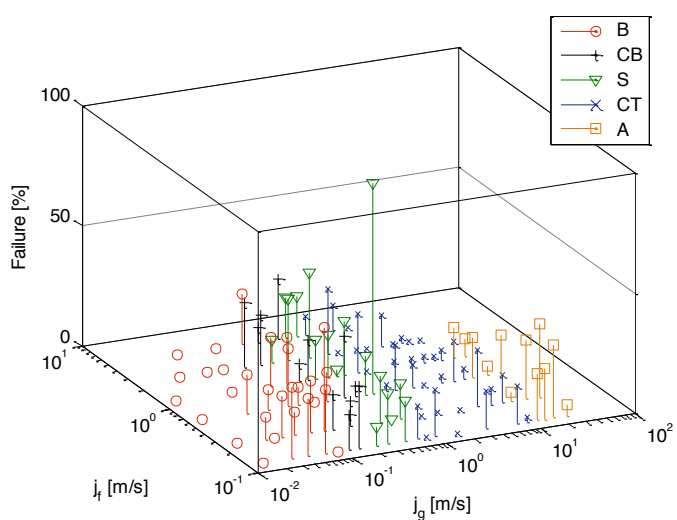

b)

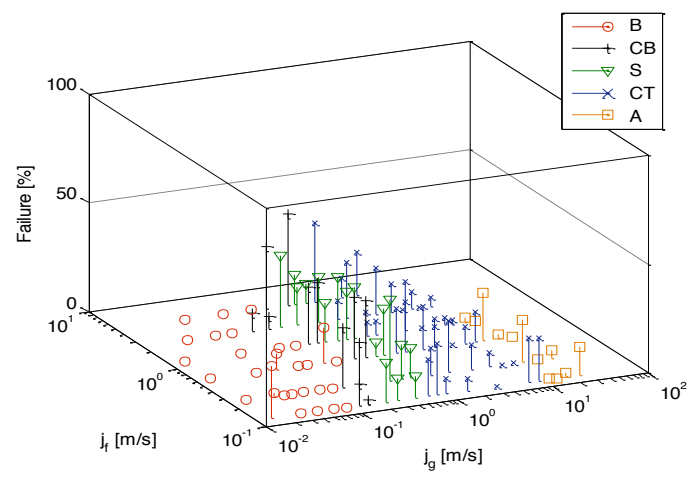

d)

Figure 12. Flow maps with the percentage of identification failures of the PNN with processing time of 1 second using impedance probe signals and a) Mean+std, b) $S_{a}, c$ ) 4IND and d) PCA as flow regime indicators 


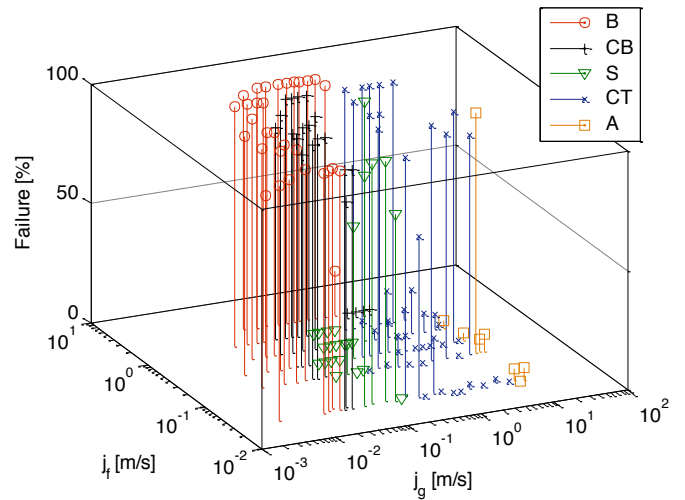

a)

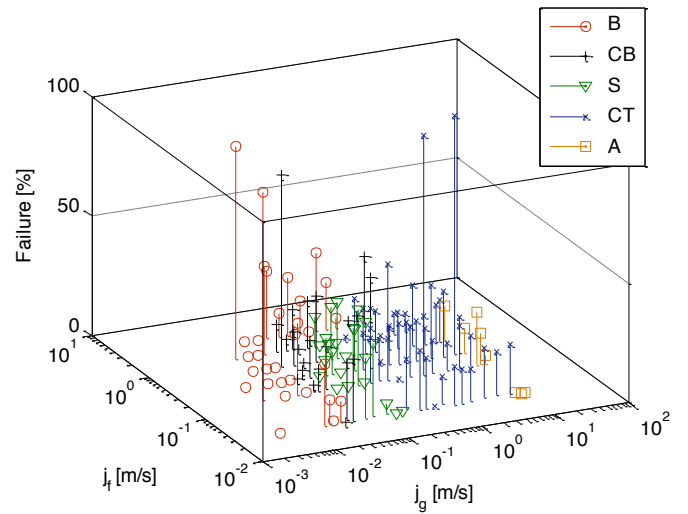

c)

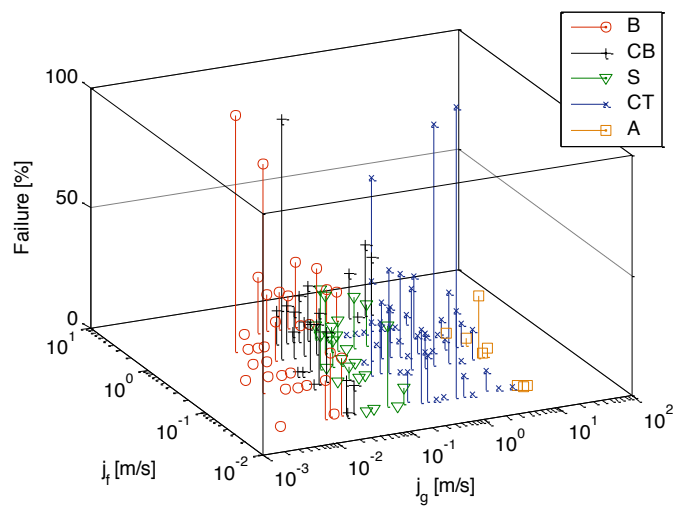

b)

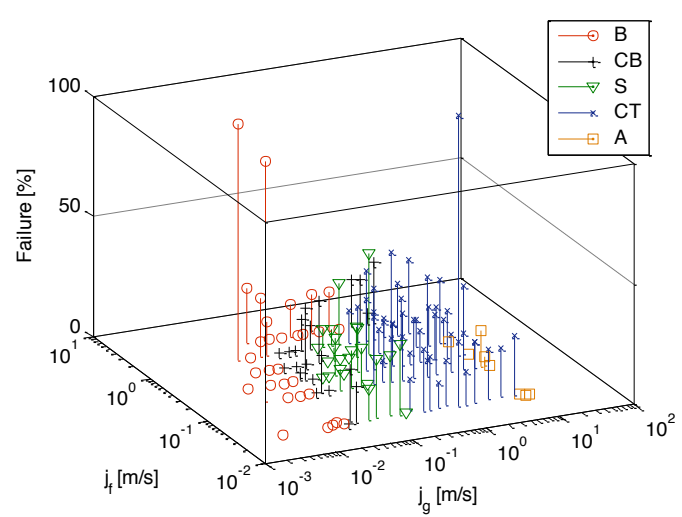

d)

Figure 13. Flow maps with the percentage of identification failures of the PNN with processing time of 1 second using conductivity probe signals and a) Mean+std, b) $S_{B}, c$ ) 4IND and d) PCA as flow regime indicators 
Table 1. Flow regime identification results for area-averaged void fraction (\% indicates agreement between identification by ANN and visual classification)

\begin{tabular}{ccccc}
\hline $\begin{array}{c}\text { Processing } \\
\text { Time [s] }\end{array}$ & S $\boldsymbol{\alpha}$ & PCA & 4IND & Mean+std \\
\hline $\begin{array}{c}\text { Number of } \\
\text { Input } \\
\text { Components }\end{array}$ & $\mathbf{1}$ & $\mathbf{8 - 1 0}$ & $\mathbf{4}$ & $\mathbf{2}$ \\
\hline 30 & $96.33 \%$ & $100.0 \%$ & $99.08 \%$ & $63.30 \%$ \\
10 & $100.0 \%$ & $100.0 \%$ & $100.0 \%$ & $60.55 \%$ \\
2 & $100.0 \%$ & $100.0 \%$ & $100.0 \%$ & $57.80 \%$ \\
1 & $99.08 \%$ & $100.0 \%$ & $99.08 \%$ & $58.72 \%$ \\
0.1 & $97.25 \%$ & $100.0 \%$ & $97.25 \%$ & $58.72 \%$ \\
\hline
\end{tabular}


Table 2. Flow regime identification results for bubble chord length indicators (\% indicates agreement between identification by ANN and visual classification).

\begin{tabular}{ccccc}
\hline $\begin{array}{c}\text { Processing } \\
\text { Time [s] }\end{array}$ & $\mathbf{S}_{\mathrm{B}}$ & PCA & 4IND & Mean+std \\
\hline $\begin{array}{c}\text { Number of } \\
\text { Input } \\
\text { Components }\end{array}$ & $\mathbf{1}$ & $\mathbf{6 - 1 0}$ & $\mathbf{4}$ & $\mathbf{2}$ \\
\hline 30 & $96.99 \%$ & $98.50 \%$ & $94.74 \%$ & $55.64 \%$ \\
10 & $96.24 \%$ & $99.24 \%$ & $98.50 \%$ & $51.13 \%$ \\
2 & $93.98 \%$ & $96.24 \%$ & $93.23 \%$ & $53.38 \%$ \\
1 & $95.49 \%$ & $97.74 \%$ & $96.24 \%$ & $48.12 \%$ \\
0.1 & $64.66 \%$ & $44.36 \%$ & $68.42 \%$ & $40.60 \%$ \\
\hline
\end{tabular}

